\title{
La representación de los europeos: sobre las posibilidades y límites de la parlamentarización de la Unión Europea*
}

\author{
The Representation of the Europeans: On the possibilities and limits \\ of the European Union parliamentarization
}

\author{
Daniel Innerarity \\ Catedrático de Filosofía Política e investigador Ikerbasque en la UPV/EHU \\ dinner@ikerbasque.org
}

doi: http://dx.doi.org/10.18543/ced-56-2017pp163-179

Sumario: I. La Europa de los parlamentos. 1. La tesis de la desparlamentarización. 2. La implicación de los parlamentos nacionales. 3. El fortalecimiento del Parlamento Europeo. 4. ¿Parlamentarización o innovación representativa?

Resumen: La Unión Europea constituye un espacio especialmente denso desde el punto de vista parlamentario y de una gran complejidad en relación con su naturaleza representativa. Para hacer frente a la desparlamentarización de sus instituciones se han propuesto dos estrategias cuyos alcances se analizan aquí: la implicación de los Parlamentos nacionales en la gobernanza europea y el fortalecimiento del Parlamento Europeo. En este artículo se examinan críticamente ambas propuestas. Para mejorar la representación en Europa se propone indagar las posibilidades de innovación representativa que permite una entidad política tan peculiar.

Palabras clave: democracia, representación, Parlamento Europeo, desparlamentarización.

Abstract: The European Union constitutes an especially dense space from the parliamentary point of view and of considerable complexity in relation with its representative nature. In order to address the de-parliamentarization of its institutions two strategies have been proposed whose outreach is here analysed: the implication of national Parliaments in the European governance and the strengthening of the European Parliament. In this article both proposals are examined critically. To improve representation in Europe, this article aims to explore the possibilities of representative innovation that such a peculiar political entity allows.

Keywords: democracy, representation, European Parliament, de-parliamentarization.

* Recibido el 15 de noviembre de 2016, aceptado el 8 de febrero de 2017. 
A partir de los años 2000 se puede constatar un «representation turn» 1 en los estudios europeos y la cuestión del déficit democrático se plantea menos como una cuestión de procedimientos de participación democrática que como un asunto relativo a la calidad de la representación. De entrada, acerca de cómo articular los múltiples modos de la representación en la Unión Europea: de los ciudadanos directamente o a través de los estados a los que pertenecen, la representación que ejercen el Parlamento Europeo y los parlamentos de los estados miembros, o de qué manera representar a los actores no estatales, los intereses de la sociedad civil, la inteligencia distribuida o la imparcialidad de las instituciones no mayoritarias. ${ }^{2}$

El Tratado de Lisboa distingue entre representación electoral, territorial, funcional y un canal directo de representación, aunque no precisa las relaciones entre ellos. Se piensa en dos tipos de subjetividad - los individuos y los estados - a los que corresponde un diferente tipo de representación: la soberanía popular y la soberanía de los estados. Si la primera apunta a una polity europea integrada, la segunda considera a la UE como una organización intergubernamental avanzada. La primera se hace valer a través de la representación electoral, funcional y directa, mientras que la segunda lo hace mediante la representación territorial. La múltiple representación que articulan las instituciones europeas obedece al intento de garantizar la presencia de todos los elementos que forman parte de ellas, tratando de evitar que sus políticas sean capturadas por los estados o por los expertos, que un nivel se imponga injustificadamente sobre otro, que se excluya a las minorías o que se prive de voz a los ciudadanos de otros estados afectados por las decisiones domésticas.

Desde una cierta perspectiva, los votantes y los políticos están actualmente confrontados a una cacofonía de pretensiones de representación, pero, desde otro punto de vista, esta pluralidad de «representation claims» puede ser entendida como una oportunidad de enriquecer la representación democrática. La integración europea plantea unos considerables desafíos a la representación política. El Tratado de Lisboa establece en su artículo 10 que el funcionamiento de la Unión debe fundarse en la democracia representativa, pero no sabemos exactamente qué significa este principio más allá del estado nación. Por si fuera poco, esta cacofonía tiene lugar en un momento de crisis de la democracia representativa, cuando la política está enfrentada cada vez más a la emergencia de poderosos actores transnacio-

${ }^{1}$ Richard Bellamy y Sandra Kröger, «Representation Deficits and Surpluses in EU Policy-Making». Journal of European Integration, 35/5, (2013): 477-497.

2 Tatjiana Evas, Ulrike Liebert y Christopher Lord, Multilayered Representation in the European Union. Parliaments, Courts and the Public Sphere, (Baden Baden: Nomos, 2012). 
nales y áreas de decisión que escapan del alcance de las tradicionales instituciones representativas.

Estos tres factores - pluralidad, novedad y crisis de la representaciónestán exigiendo un salto conceptual a la hora de entender y diseñar la representación. Un ejemplo negativo de falta de innovación conceptual lo encontramos en la sentencia del Tribunal Constitucional alemán sobre el Tratado de Lisboa, cuando aboga por optar entre una plena parlamentarización de la UE o por fortalecer los parlamentos nacionales; de este modo deja de tomar en consideración las nuevas posibilidades de concebir de la relación representativa. Parece así no terminar de entender que el gran reto intelectual y práctico que tenemos es liberar la noción de representación del vínculo que la asociaba tan fuertemente al territorio y al demos. Los procesos de europeización y globalización plantean enormes desafíos: ya no existe la clara demarcación de los demoi representados, no hay un claro centro de autoridad institucional y el derecho a votar ha perdido su simplicidad como medida de la igualdad política. La tarea que debemos acometer es imaginar cómo volver a pensar las relaciones representativas en medio de tales contextos.

\section{La Europa de los parlamentos}

El Tratado de Lisboa ha sido llamado el «tratado de los parlamentos» ${ }^{3}$ porque introduce muchas consideraciones acerca de la democracia representativa y la función que en ella ejercen los parlamentos, instando a la colaboración entre el Parlamento Europeo y los parlamentos de los estados miembros. Esta declaración no ha servido para desactivar la denuncia de que asistimos - en general, pero muy en particular en la Unión Europea - a un progresivo debilitamiento de los parlamentos, proceso frente al que se ha propuesto la inclusión de los parlamentos de los estados miembros en el decisión-making europeo así como incrementar las competencias del Parlamento Europeo. Mi hipótesis es que las posibilidades de reparlamentarización de la UE son limitadas, pero hay diversas posibilidades de pensar de una manera innovadora las funciones representativas.

${ }^{3}$ Elmar Brok y Martin Selmayr, «Der 'Vertrag der Parlamente' als Gefahr für die Demokratie? Zu den offensichtlich unbegründeten Verfassungsklagen gegen den Vertrag von Lissabon» en Integration 3, (2008): 217-234. 


\section{La tesis de la desparlamentarización}

La denuncia de una progresiva irrelevancia de los parlamentos frente a los poderes ejecutivos es una crítica general en todas las democracias, que parece más verosímil aún en la Unión Europea debido a las peculiaridades de la integración. El parlamentarismo se debilita tanto a nivel nacional como en un parlamentarismo europeo incompleto. Tanto en un caso como en otro, los gobiernos dictan la agenda a los parlamentos y no al revés. ${ }^{4}$ En la Unión Europea se constata una captura de los poderes de decisión por parte de las instituciones ejecutivas (Comisión Europea y estados miembros), cuyo resultado es un debilitamiento de los poderes legislativos $\mathrm{y}$, en consecuencia, de los electorados que los eligen. ${ }^{5}$ Los poderes ejecutivos se constituyen así como los "gatekeepers» de la política europea. ${ }^{6}$ En el transcurso de la integración europea los parlamentos nacionales han perdido competencias legislativas; conservan un cierto control sobre los cambios en materia de tratados (al menos cuando la ratificación no tiene lugar mediante referéndum), pero no participan directamente en las negociaciones europeas. Los parlamentos nacionales carecen de control sobre la política europea, sufren una falta de autoridad sobre las políticas transnacionales y se convierten en los principales perdedores de la integración europea. Al mismo tiempo la integración europea ha provocado un desplazamiento de poder hacia los ejecutivos nacionales. No parece exagerado afirmar que la desparlamentarización es el elemento más preocupante de la democracia en Europa. ${ }^{7}$

La gestión de la crisis económica y del euro no ha mejorado en absoluto la situación. Por la vía de la informalidad y las estructuras ad hoc, Europa

${ }^{4}$ Carina Sprungk, «Parlamentarismus in europäischen Mehrebenensystem. Zum Wandel von Rollenanforderungen an nationale Parlamente in EU Angelegenheiten», en Eppler Abels (eds.), Auf dem Weg zum Mehrebenenparlamentarismis? Funktionen von Parlamenten im politischen System der EU, (Baden-Baden: Nomos, 2011): 211-226.

5 Katrin Auel y Arthur Benz, «The politics of adaptation: the Europeanisation of national parliamentary systems» en The Journal of Legislative Studies 11, 3/4, (2005): 373; Tapio Raunio y Simon Hix, «Backbenchers learn to fight back: European integration and parliamentary government»en West European Politics 23/4, (2000): 142-168.

6 Vivien Schmidt, «European 'federalism' and its encroachments on national institutions» en Publius 29, Nº1, (1999): 19-44; Andreas Maurer y Wolfgang Wessels, (eds.) National Parliaments on their Ways to Europe: Losers or Latecomers? (Baden-Baden: Nomos, 2001); Richard Bellamy y Sandra Kröger, «Domesticating the democratic deficit? The role of national parliaments and parties in the EU's system of governance» en Parliamentary Affairs, 67 / 2, (2012): 437-457.

7 Johannes Pollak, «Compounded Representation in the EU: No Country for Old Parliaments?», en Sandra Kröger, (ed), Political Representation in the European Union: Still Democratic in Times of Crisis?, (London/New York: Routledge, 2014): 37-69. 
ha tratado de responder a la crisis profundizando más en la división entre el espacio de los procedimientos democráticos y el espacio de la decisión política. La crisis ha puesto de manifiesto la enorme debilidad parlamentaria, ya que los controles parlamentarios apenas podían llevarse a cabo en aquellos ámbitos en los que se decidía intergubernamentalmente y que remitían finalmente a los niveles nacionales, como pusieron en evidencia los mecanismos de rescate de 2010 y 2012, el European Financial Stability Facility (EFSF), al principio, y el European Stability Mechanism (ESM), después.

Este diagnóstico requeriría no pocos matices, pero trataré de posponer mi punto de vista para cuando examinemos los límites de la parlamentarización de la Unión Europea y las otras posibles innovaciones representativas que deberíamos considerar. De momento nos toca abordar las dos estrategias que se han propuesto para revertir esta situación: asegurar la presencia de los parlamentos nacionales en el decision-making europeo y fortalecer el Parlamento Europeo. En el primer caso, se trataría de subrayar el enraizamiento nacional de la democracia en Europa y en otro de europeizar la democracia parlamentaria.

\section{La implicación de los parlamentos nacionales}

La previsión que anima esta propuesta es que una mayor intervención de los actuales parlamentos contribuiría a aumentar la legitimidad democrática de la Unión. Esta previsión se apuntaba en aquella apelación del Tratado de Maastricht (1992) a una mayor colaboración entre los parlamentos (todos los cuales tienen sus Comisiones de Asuntos Europeos y se reúnen en la Conférence des organes parlamentaires spécialisés dans les affaires de l'Union dos veces al año desde 1989), así como en el «Protocol on the role of National Parliaments in the European Union» añadido al Tratado de Amsterdam (1997), pero quien lo plantea abiertamente es el Tratado de Lisboa, que marcó un paso en este sentido unos años más tarde (2007); no sólo es el primero que menciona a los parlamentos nacionales en el texto principal sino que les confiere un estatuto potencial muy significativo en la gobernanza democrática de la Unión Europea.

La nueva estructura del Tratado de Lisboa ayudó a que los parlamentos nacionales dejaran de ser meras «víctimas de la integración» y se constituyeran en agentes protagonistas. ${ }^{8}$ Las enmiendas introducidas en el Tratado

${ }^{8}$ Karlheinz Neunreither, «The Democratic Deficit of the European Union: Towards Closer Cooperation between the European Parliament and the National Parliaments»en Government and Opposition 29/3, (1994): 299-314; Karlheinz Neunreither, «The European Parliament and National Parliaments: Conflict or Cooperation?» en Katrin Auel y Arthur 
de Lisboa implican a los parlamentos nacionales como guardianes de la subsidiaridad en el proceso legislativo europeo. ${ }^{9}$ Se les confiere la función de «alarma temprana» para vigilar que se cumpla el principio de subsidiaridad..$^{10} \mathrm{El}$ alcance de estas previsiones es discutido. Mientras que, por ejemplo, para Rittberger ${ }^{11}$ esto no supone otra cosa que complementar las funciones legitimadoras del Parlamento Europeo, para Lindseth ${ }^{12}$, es parte de un esfuerzo por reconectar la gobernanza europea con la democracia representativa a nivel nacional. En cualquier caso, aunque estos poderes que se otrogan a los parlamentos son más bien limitados y excepcionales, de este modo quedaría establecido lo que algunos han llamado «Multilevel Parliamentary Field» ${ }^{13}$, un espacio de interacción entre los parlamentos que comparten la tarea de representar a los europeos y las europeas en el proceso de decisión de la Unión.

Ahora bien, muchos investigadores son más bien escépticos en relación con el Early Warning System. En primer lugar porque el sistema ignora el hecho de que raramente la mayoría parlamentaria adopta una posición diferente del gobierno. En los estados miembros la división entre gobierno y oposición es el principal cleavage, no la división entre gobierno y mayoría parlamentaria. Por esta razón es infrecuente que el gobierno y la mayoría parlamentaria tengan una posición diferente, de manera que no es verosímil que en un parlamento de un estado se plantee una posición diferente de la que su gobierno mantiene en el Consejo.

Por otro lado, cabría advertir de que esta práctica supondría un debilitamiento de la capacidad deliberativa a nivel europeo. En la literatura que analiza los derechos de co-decisión de los parlamentos frente a sus ejecutivos se pone como ejemplo al Folketing danés, con capacidad para dar un

Benz (eds.), The Europeanisation of Parliamentary Democracy, (London: Routledge, 2006): 164-187; Andreas Maurer y Wolfgang Wessels, (eds.) National Parliaments on their Ways to Europe: Losers or Latecomers?, (Baden-Baden: Nomos, 2001); J. O'Brennan y Tapio Raunio, (eds.) National Parliaments within the Enlarged European Union: From 'Victims' of Integration to Competitive Actors?, (London/New York: Routledge, 2007).

${ }^{9}$ Christian Calliess, Die neue Europäische Union nach dem Vertrag von Lissabon. Ein Überblick über die Reformen unter Berücksichtigung ihrer Implikationen für das deutsche Recht, (Tübingen: Mohr Siebeck, 2010).

${ }^{10}$ Ian Cooper, «The Watchdogs of Subsidiarity: National Parliaments and the Logic of Arguing in the EU»en Journal of Common Market Studies 44 (2), (2006): 281-304.

11 Berthold Rittberger, Building Europe's Parliament. Democratic Representation Beyond the Nation State, (Oxford University Press: 2005).

12 Peter Lindseth, Power and Legitimacy: Reconciling Europe and the Nation-State, Oxford University Press, 2010.

13 Ben Crum y John Erik Fossum, «The Multilevel Parliamentary Field - A Framework for Theorising Representative Democracy in the EU» en European Political Science Review 1, (2009): 249-271. 
mandato vinculante a su gobierno para las negociaciones. Por lo general, los parlamentos nacionales únicamente tienen la capacidad de adoptar resoluciones no vinculantes acerca de asuntos europeos (excepto en Dinamarca y Austria, donde son vinculantes). Aunque la práctica del Parlamento de Dinamarca es encomiable en lo que tiene de responsabilidad y control sobre los asuntos europeos, su mandato imperativo impide la deliberación abierta sobre sus decisiones en el seno de la Unión Europea. La parlamentarización de la Unión Europea no contribuye necesariamente a democratizarla en el sentido de hacerla más deliberativa. Fortalecer el papel de los parlamentos nacionales puede condicionar la posición de los gobiernos de modo que condicionen su posición negociadiora y no les permitan margen de maniobra. ${ }^{14} \mathrm{El}$ entramado institucional de la Unión quedaría más vinculado con los electorados nacionales pero menos predispuesto a entendimientos transnacionales. La incorporación de los parlamentos nacionales equivaldría a incrementar la legitimidad nacional cuando lo que hace falta es mayor legitimidad a nivel europeo. No necesitamos agregación parlamentaria sino emergencia parlamentaria; la solución no es conectar con los electorados que ya tenemos sino con las lógicas transnacionales que deben ir surgiendo de nuestras prácticas. Para impulsar una mayor integración, los parlamentos no deben solo pensar en líneas rojas, subsidiaridad e intereses específicos, sino también en términos de cómo pueden contribuir positivamente al proceso de integración europea.

Hay otra razón de esta limitada viabilidad de implicar a los parlamentos en los grandes asuntos de la Unión, una razón que tiene carácter funcional, de hábitos y cultura política. Los parlamentos de los estados no están preparados para internalizar las preocupaciones de los otros estados ni articular una forma de solidaridad entre ellos. A diferencia de los jefes de gobierno, los parlamentarios no están acostumbrados a justificar las decisiones adoptadas a nivel europeo ante sus propios electorados. «Una mayor presencia de las cuestiones de ámbito europeo en los parlamentos nacionales únicamente removería la competición entre los partidos nacionales sin ninguna fuerza estructural de contrapeso que indujera a una consideración justa de los intereses del resto de la población europea». ${ }^{15}$

Si es que hay un déficit de representación a nivel europeo, implicar más a los parlamentos nacionales no mejoraría las cosas y tampoco es verosímil que esta capacitación vaya a darles un mayor protagonismo, por lo que no

${ }^{14}$ Katrin Auel y Arthur Benz, «A Disputed Idea Becomes Law: Remarks on European Democracy as a legal Principle», en Beate Kohler-Koch, Berthold Rittberger, (eds.), Debating the Democratic Legitimacy of the European Union, Plymouth: Rowman, 2007: 33-44.

15 Philippe Van Parijs, «Justifying Europe», en Luuk van Middelaar y Philippe Van Parijs (2015), After the storm. How to save democracy in Europe, (Tielt: Lannoo, 2015): 256. 
es exagerado afirmar que seguirán siendo unos actores bastante débiles en el sistema político de la Unión ${ }^{16}$.

\section{El fortalecimiento del Parlamento Europeo}

El Parlamento Europeo ha evolucionado desde un cuerpo consultativo y no elegido a una de las asambles más poderosas del mundo, más que muchos parlamentos nacionales en Europa, y el único parlamento supranacional del mundo. Con la decisión de erigir en 1976 un Parlamento Europeo mediante sufragio directo y universal, se crea una institución que pretende articular la voluntad general europea no como una mera agregación de intereses nacionales o equilibrios institucionales sino a un nivel genuinamente supranacional. Desde 1979 es elegido directamente por los ciudadanos; es co-legislador con el Consejo en un amplio número de cuestiones y puede exigir cuentas a la Comisión. Es evidente que esto no ha convertido a la Unión Europea en una democracia representativa «fullfledged», pero ha incrementado su carácter parlamentario. No deberíamos subestimar, por tanto, el actual grado de poder del Parlamento Europeo, pese a que parecen razonables muchas de las propuestas de fortalecer sus poderes.

Algunas críticas al déficit democrático se refieren al papel limitado del Parlamento Europeo en virtud de que buena parte de las decisiones han sido transferidas a instituciones solo indirectamente representativas (como el Consejo). No son pocos los que reclaman dar más poder al Parlamento Europeo, entendiendo que así se fortalecería el vínculo entre los ciudadanos y sus representantes, hasta el punto de que las elecciones europeas podrían convertirse en $«$ first-order elections». ${ }^{17}$ En esta línea apunta también la propuesta de elección del presidente de la Comisión por los miembros del Parlamento Europeo, que fue introducida por primera vez en 2014. ${ }^{18}$ También

16 Tapio Raunio, «National Parliaments and the Future of European Integration: Learning to Play the Multilevel Game» en Achim Hurrelmann, Steffen Schneider y Jens Steffek (eds.), Legitimacy in an Age of Global Politics, New York: Palgrave Macmillan, (2007): 158-173.

17 Andreas Follesdal y Simon Hix, «Why there is a democratic deficit in the EU: A response to Majone and Moravcsik» en Journal of Common Market Studies 44,3, (2006): 533-562.

18 Frank Decker, Governance beyond the nation-state Reflections on the democratic deficit of the European Union. Journal of European Public Policy 9/2, (2002): 256-272. Simon Hix, Why the EU should have a single president, and how she should be elected. Paper for the working group on democracy in the EU for the UK Cabinet Office, Oct. 2002, http://personal.lse.ac.uk/hix/Working_Papers/Why\%20the\%20EU\%20Should\%20Have\%20 a\%20Single\%20Pres-ident.pdf, acceso el 22 de enero de 2013. 
suelen atribuirse las dificultades para desarrollar la democracia en la Unión Europea a la falta de un sistema de partidos a nivel europeo capaz de generar grupos de gobierno y oposición dentro del PE. ${ }^{19}$ Quienes así piensan argumentan que incrementando la competición política a nivel europeo a través del empoderamiento del Parlamento Europeo los votantes estarán más interesados en participar en las elecciones y se disolvería el déficit democrático.

Este análisis es muy cuestionable. La transferencia continua de competencias al Parlamento Europeo ha ido acompañado de una disminución de la participación en dichas elecciones.$^{20}$ Los poderes del Parlamento Europeo no han dejado de crecer, por ejemplo, en las revisiones de Amsterdam (1997) y de Niza (2001), a pesar de lo cual la participación electoral no ha aumentado; parece incluso haberlo hecho en proporción inversa al incremento de sus competencias.

Por otro lado, este fortalecimiento del Parlamento Europeo podría producir un déficit de representación de los miembros más pequeños, a los que protege el actual sistema. ${ }^{21} \mathrm{El}$ fortalecimiento del Parlamento Europeo, que no es un organismo federal sino central, tendría efectos centralizadores. ${ }^{22}$ Teniendo presente esta posibilidad, la solución de la Convención Europea consistió en fortalecer todas las instituciones de la Unión sin alterar significativamente el equilibrio entre ellas. El hecho de que la representación en el Parlamento Europeo no siga estrictamente el criterio de la población responde a su peculiaridad institucional. La Unión Europea difiere de los estados federales en que su desigual representación no está confinada en una segunda cámara sino en todos sus órganos, desde el Parlamento hasta el Consejo. La diferencia y no la igualdad es el principio organizador de la Unión Europea, lo que no es un déficit sino el resultado de una historia y un énfasis en la protección de los pequeños estados contra la dominación de los grandes.

19 Andreas Follesdal y Simon Hix, «Why there is a democratic deficit in the EU: A response to Majone and Moravcsik» en Journal of Common Market Studies 44,3, (2006): 533-562.

${ }^{20}$ Joseph Weiler, «In the face of crisis: input legitimacy, output legitimacy and the political messianism of European integration» en Journal of European Integration 34/7, (2012): 830 .

${ }^{21}$ Renaud Dehousse, «Constitutional reform in the European Community: are there alternatives to the majoritarian avenue?» en Jack Hayward (ed.), The Crisis of Representation in Europe, London: Frank Cass, (1995): 118; Vivien Schmidt, Democracy in Europe. The EU and National Polities, Oxford University Press, (2006): 273.

22 Dieter Grimm, «Treaty or Constitution? The legal basis of the European Union after Maastricht», in E. Eriksen, J.E. Fossum and A.J. Menéndez (eds.), Developing a Constitution for Europe, London: Routledge, (2004): 69-87. 
Es muy cuestionable que la democratización de la Unión Europea venga de la mano del Parlamento Europeo como su principal actor. Por mucho que se atribuyan al Parlamento mayores poderes, no se creará con ello un demos o una espacio público unificado. Para resolver democráticamente el problema de la representación en Europa se requiere una estructura diferenciada de instituciones y procedimientos. Los poderes del Parlamento Europeo solo pueden hacerse valer con un alto nivel de consenso y con un acuerdo interinstitucional del Consejo y la Comisión. Estos condicionamientos no responden a una tímida democratización sino a la realidad de una complejidad democrática.

Seguramente habrá que dotar de mayores competencias al Parlamento Europeo, como la capacidad de enmendar todas las áreas del presupuesto o extender el «Qualified Majority Vote» a ámbitos fiscales; también ha sido muy positivo que desde 2014 el presidente de la Comisión sea elegido por el Parlamento en vez de por el Consejo. De ese modo puede haber más batalla política y movilización en torno a las decisiones europeas. También pueden mejorarse sus funciones de control. Por ejemplo: aunque el Tratado de Lisboa plantea que las recomendaciones de política fiscal hacia un país en concreto sean llevadas a cabo por el Consejo únicamente, nada prohíbe implicar al Parlamento europeo en dicha supervisión, a nivel de plenario o de comité, lo que estaría más justificado cuando esto implica una modificación del presupuesto europeo. ${ }^{23}$ Incluso en aquellas cuestiones en las que el Parlamento Europeo no decide, sí que puede adoptar mayores capacidades de supervisión y control sobre las instituciones ejecutivas. Una mayor $a c$ countability del Consejo Europeo y del Eurogrupo antes y después de sus respectivas Cumbres contribuiría a corregir la deriva de la Unión hacia un poder ejecutivo excesivo. ${ }^{24}$

Pero no pensemos que esos procedimientos van a fortalecer mágicamente la identificación de los ciudadanos con Europa porque la hacen algo más similar a lo que conocemos en el nivel nacional. Además de esta democratización por analogía con lo conocido, es necesario una forma de democratización en consonancia con la peculiaridad de la Unión que ninguna similitud con la lógica nacional cubrirá completamente.

${ }^{23}$ Mark Dawson, «The Euro Crisis and Its Transformation of the EU Law and Politics» en The Governance Report 2015, Hertie School of Governance, Oxford University Press, (2015): 41-68.

${ }^{24}$ Sonia Piedrafita y Steven Blockmans, Shifting EU Institutional Reform into High Gear: Report of the CEPS High-Level Group, (Brussels: Centre for European Policy Studies, 2014): 20 . 


\section{4. ¿Parlamentarización o innovación representativa?}

La extendida frustración pública en relación a la democracia europea no se superará aumentando las competencias del Parlamento Europeo, añadiendo mecanismos de transparencia o cualquier otro procedimiento de ingeniería institucional, sino cuando acertemos a entender su naturaleza compleja y dejemos de medirla con unos estándares que nunca podrá satisfacer. «Para los gobiernos ha sido extremadamente difícil resistirse a incrementar el papel del Parlamento Europeo porque no han sido capaces de formular una alternativa para resolver el "déficit democrático"». ${ }^{25} \mathrm{El}$ hecho de que no hayamos pensado bien las deficiencias de la democracia en Europa al mismo tiempo que sus potencialidades específicas explica la recurrente falta de originalidad con la que abordamos el asunto y que no haya otras propuestas que introducir procedimientos democráticos convencionales de los estados nacionales al nivel supranacional. Lo hacemos además sin caer en la cuenta de que los procedimientos y las instituciones que parecen más democráticas en un contexto pueden ser inapropiadas en otro..$^{26}$

Pensemos, por ejemplo, en la estrategia de dar a las instituciones europeas el mismo nombre que las instituciones nacionales y dotarlas así de un aire de analogía y familiaridad con lo ya conocido. De este modo esquivamos sus características específicas y parecemos desconocer que nos encontramos ante instituciones que se rigen de acuerdo con principios diferentes y que cumplen funciones diferentes. La homonimia es una estrategia recurrente pero poco útil cuando se adentra uno en terreno ignoto y suele terminar generando mayores perplejidades. Este es el caso del Parlamento Europeo, que difiere de los parlamentos nacionales no solo porque carece de la capacidad de iniciativa legislativa, en materia fiscal y de gasto o por la poca presencia del antagonismo típico entre gobierno y oposición. Su naturaleza sui generis también se pone de manifiesto en la actitud de sus miembros, muchos de los cuales se consideran más especialistas en determinadas materias que políticos de partido, o en que se exija de la Comisión Europea una cierta neutralidad, y esto es precisamente lo que explica en parte que nunca se haya contestado seriamente su monopolio de iniciativa legislativa. ¿Por qué insistir en que el Parlamento Europeo se parezca más a los parlamentos conocidos y no indagar si en sus peculiaridades, más que una carencia, lo que se manifiesta es una función inédita sobre la que deberíamos pensar y tal vez potenciar? Sería algo así como una política más allá de las

25 Michael Shackleton, «The European Parliament», en John Peterson y Michael Shackleton, The Institutions of the European Union, Oxford University Press, (2012): 145.

${ }^{26}$ William Nelson, «The Institutions of Deliberative Democracy» en Social Philosophy \& Policy 17, (2000): 181-202. 
formas clásicas de antagonismo y un Parlamento cuya función representativa es más importante que la de controlar al poder ejecutivo.

Una de las propiedades que más inquietud suscita entre algunos es el hecho de que en el Parlamento Europeo haya tan poco antagonismo ideológico y que la división entre gobierno y oposición juegue un menor papel que en los parlamentos conocidos. Ahora bien, ¿por qué lo sustancial de la política tiene que ser la batalla por el control del poder y la rivalidad? ¿No puede haber otras formas de hacer política más consensuales y menos agonísticas en aquellos espacios que, como la Unión Europea, son más complejos y tienen mayores exigencias de integración? La dimensión consensual de la política europea tiene razones funcionales en una polity de cohesión escasa. Si una mayoría tuviera más poder en Parlamento habría más en juego en las elecciones europeas y tal vez el electorado se movilizaría más, pero la desafección hacia la Unión aumentaría en la minoría perdedora, que podría no sentirse representada y desidentificarse. Se afirma con frecuencia que el espíritu de consenso empuja a los discrepantes a cuestionar las policies rechazando la polity, es decir, en forma de desafección hacia el proyecto europeo, pero pensemos si no sería peor el efecto disgregador si se instaurara una lógica estrictamente mayoritaria que podría suscitar los mismos desafectos en los eventuales perdedores (mayores en número que cuando rige una lógica consensual).

Podemos considerar como ventajas o astucias funcionales lo que comparado con aquello que conocemos se nos presenta de entrada como un defecto. Dado que la división gobierno-oposición no tiene una importancia decisiva en la gobernanza europea, la deliberación está obligada a jugar un mayor papel que en los parlamentos nacionales. ${ }^{27}$ Los poderes de control del Parlamento Europeo son ciertamente limitados, pero deberían ser puestos en el contexto de un sistema semi-presidencialista, en el que el ejecutivo (la Comisión) no es un gobierno de partido y el Parlamento Europeo, más que una institución de debate es un «working parliament». ${ }^{28}$ La Unión Europea no es una democracia mayoritaria imperfecta sino una forma de democracia compleja que limita intencionalmente la aplicación de los principios mayoritarios. ${ }^{29}$ En virtud de sus propiedades específicas, el Parlamento

27 Kyosti Pekonen, «Members of the European Parliament as Delegates and Parliamentarians», en Claudia Wiesner, Kari Palonen y Tapani Turkka (eds.), Parliament and Europe. Rhetorical and conceptual studies on their contemporary connections, Baden-Baden: Nomos, (2011): 59-78.

${ }_{28}$ Teija Tiilikainen, «The Role of the European Parliament in the EU's Political Order» en Claudia Wiesner, Kari Palonen y Tapani Turkka (eds.), Parliament and Europe. Rhetorical and conceptual studies on their contemporary connections, (Baden-Baden: Nomos, 2011): 39.

29 Jürgen Neyer, The Justification..., 2012, 161. 
Europeo tiene una oportunidad de estar más cerca de ciertos principios parlamentarios que los parlamentos nacionales.

Volvamos a la tesis de la desparlamentarización, que se apoyaba fundamentalmente en su escaso poder de control sobre el gobierno (en este caso, la Comisión Europea). Podríamos comenzar recordando que tampoco los parlamentos nacionales controlan demasiado a sus gobiernos, aunque sea por distintas razones. La función del Parlamento Europeo en la gobernanza europea debe pensarse en consonancia con la evolución de las instituciones parlamentarias en general, que distan mucho de ser el instrumento con el que los estados pretendían controlar a sus gobiernos y dotarse de iniciativa legislativa.

Los parlamentos tienen la doble función de representación y de control del gobierno, pero esta doble función no tiene porqué ser replicada a nivel europeo. En Europa hay una democracia representativa más que un gobierno representativo. ${ }^{30}$ El Parlamento Europeo representa a los ciudadanos pero no controla las acciones del gobierno. Hay una representación sin conexión con un gobierno. Pero cabe pensar que un parlamento liberado de la función de sostener a un ejecutivo puede desempeñar una función de escrutinio más independiente que los parlamentos nacionales, lo que puede fortalecer la democracia representativa en Europa.

Es cierto que para que un poder ejecutivo sea algo diferente de la mera administración, el legislativo debe poder controlar de algún modo al ejecutivo, lo que en el caso de la Unión Europea es muy limitado. No es verosímil que la Unión evolucione hacia una democracia plenamente parlamentaria, pero el control sobre el ejecutivo por parte de diversos actores - entre ellos, el Parlamento Europeo - puede ser mejorado y ampliado con los criterios de buena gobernanza formulados en el Libro blanco de la gobernanza europea (apertura, participación, responsabilidad, efectividad y coherencia). Además, aunque el Parlamento Europeo solo tenga formalmente derechos de información y consulta, la intensidad y regularidad del debate con los cargos y altos representantes se traduce en un escrutinio de gran intensidad. ${ }^{31}$ La tarea principal del Parlamento Europeo no es representar a un Pueblo europeo que no existe como tal sino acompañar críticamente el trabajo de la Comisión y del Consejo.

La tesis de la desparlamentarización es incompleta ya que se centra excesivamente en las funciones legislativas de los parlamentos descuidando

30 Peter Mair y Jacques Thomassen, «Political Representation and Government in the European Union» en Journal of European Public Policy 17 (1), (2010): 22.

31 Daniel Thym, «Beyond Parliament's Reach? The Role of the European Parliament in the CFSP», European Foreign Affairs, 11, (2006): 119. 
otras tan importantes..$^{32}$ La función de control no lo es todo. Están también las funciones de información y las específicamente representativas, que pueden incluso llevarse a cabo mejor si están parcialmente liberados de la obligación de controlar al gobierno. El control del gobierno no es necesariamente la finalidad principal de los parlamentos sino la reducción del information gap entre los parlamentos y el gobierno y, en segundo lugar, la información de los ciudadanos.

Podemos concluir afirmando que la parlamentarización no es el único medio de asegurar la representación en la Unión Europea, aunque el potencial del Parlamento Europeo y de los otros parlamentos pueda mejorarse. Democratización de la Unión no equivale a su plena parlamentarización, lo que no es ni posible ni acorde con esa complejidad que debe ser democratizada. La Unión Europea no es un sistema de soberanía parlamentaria sino de separación de poderes. En consonancia con ello, la Comisión Europea no es un gobierno parlamentario en una democracia mayoritaria ni el ejecutivo de un sistema presidencial; es un organismo colegial que plantea no pocos quebraderos de cabeza a la teoría de la democracia, instituida para proteger el bien común europeo y como ejecutivo autónomo que debe velar para que los intereses particulares nacionales no condicionen en exceso la política europea. La representación en la Unión Europea deberá ser compleja, con equilibrios institucionales sofisticados y procesos deliberativos interconectados.

\section{Bibliografía}

Auel, Katrin. «Zur "Europatauglichkeit" mitgliedstaatlicher Demokratie - Diagnoses und Therapien der Entparlamentarisierung», en Grenzen der europäischen Integration. Herausforderungen für Recht und Politik de Claudio Franzius, Franz C. Mayer, Jürgen Neyer (eds.), Baden-Baden: Nomos, 2014: 233-249.

Auel, Katrin y Benz, Arthur. «The politics of adaptation: the Europeanisation of national parliamentary systems» en The Journal of Legislative Studies 11, 3/4, (2005): 372-393.

Auel, Katrin y Benz, Arthur. «A Disputed Idea Becomes Law: Remarks on European Democracy as a legal Principle», en Beate Kohler-Koch, Berthold Rittberger, (eds.), Debating the Democratic Legitimacy of the European Union, Plymouth: Rowman, 2007: 33-44.

${ }^{32}$ Katrin Auel, «Zur 'Europatauglichkeit' mitgliedstaatlicher Demokratie - Diagnoses und Therapien der Entparlamentarisierung», en Grenzen der europäischen Integration. Herausforderungen für Recht und Politik de Claudio Franzius, Franz C. Mayer, Jürgen Neyer (eds.), Baden-Baden: Nomos, 2014: 233-249. 
Bellamy, Richard y Kröger, Sandra. «Domesticating the democratic deficit? The role of national parliaments and parties in the EU's system of governance» en Parliamentary Affairs, 67/2, (2012): 437-457.

Bellamy, Richard y Kröger, Sandra. «Representation Deficits and Surpluses in EU Policy-Making», Journal of European Integration, 35/5, (2013): 477-497.

Brok, Elmar y Selmayr, Martin. «Der 'Vertrag der Parlamente' als Gefahr für die Demokratie? Zu den offensichtlich unbegründeten Verfassungsklagen gegen den Vertrag von Lissabon» en Integration 3, (2008): 217-234.

Calliess, Christian. Die neue Europäische Union nach dem Vertrag von Lissabon. Ein Überblick über die Reformen unter Berücksichtigung ihrer Implikationen für das deutsche Recht, Tübingen: Mohr Siebeck, 2010.

Cooper, Ian. «The Watchdogs of Subsidiarity: National Parliaments and the Logic of Arguing in the EU»en Journal of Common Market Studies 44 (2), (2006): 281-304.

Crum, Ben y Fossum, John Erik. «The Multilevel Parliamentary Field - A Framework for Theorising Representative Democracy in the EU» en European Political Science Review 1, (2009): 249-271.

Dawson, Mark. «The Euro Crisis and Its Transformation of the EU Law and Politics» en The Governance Report 2015, Hertie School of Governance, Oxford University Press, (2015): 41-68.

Decker, Frank. Governance beyond the nation-state Reflections on the democratic deficit of the European Union. Journal of European Public Policy 9/2, (2002): 256-272.

Dehousse, Renaud. «Constitutional reform in the European Community: are there alternatives to the majoritarian avenue?» en Jack Hayward (ed.), The Crisis of Representation in Europe, London: Frank Cass, (1995): 118-36.

Evas, Tatjiana, Liebert, Ulrike y Lord, Christopher. Multilayered Representation in the European Union. Parliaments, Courts and the Public Sphere, Baden Baden: Nomos, 2012.

Follesdal, Andreas y Hix, Simon. "Why there is a democratic deficit in the EU: A response to Majone and Moravcsik» en Journal of Common Market Studies 44,3, (2006): 533-562.

Grimm, Dieter. «Treaty or Constitution? The legal basis of the European Union after Maastricht», en E. Eriksen, J.E. Fossum y A.J. Menéndez (eds.), Developing a Constitution for Europe, London: Routledge, (2004): 69-87.

Hix, Simon. Why the EU should have a single president, and how she should be elected. Paper for the working group on democracy in the EU for the $U K$ Cabinet Office, Oct. 2002, http://personal.1se.ac.uk/hix/Working_Papers/ Why $\% 20$ the $\% 20$ EU\%20Should $\% 20$ Have $\% 20$ a\%20Single $\% 20$ Pres-ident.pdf, acceso el 22 de enero de 2013.

Lindseth, Peter. Power and Legitimacy: Reconciling Europe and the Nation-State, Oxford University Press, 2010.

Mair, Peter y Thomassen, Jacques. «Political Representation and Government in the European Union»en Journal of European Public Policy 17 (1), (2010): 20-35.

Maurer, Andreas y Wessels, Wolfgang (eds.). National Parliaments on their Ways to Europe: Losers or Latecomers?, Baden-Baden: Nomos, 2001. 
Nelson, William. «The Institutions of Deliberative Democracy» en Social Philosophy \& Policy 17, (2000): 181-202.

Neunreither, Karlheinz. «The Democratic Deficit of the European Union: Towards Closer Cooperation between the European Parliament and the National Parliaments»en Government and Opposition 29 / 3, (1994): 299-314.

Neunreither, Karlheinz. «The European Parliament and National Parliaments: Conflict or Cooperation?» en Katrin Auel y Arthur Benz (eds.), The Europeanisation of Parliamentary Democracy, London: Routledge, (2006): 164-187.

Neyer, Jürgen. The Justification of Europe, Oxford University Press, 2012.

O'Brennan, John y Raunio, Tapio (eds.). National Parliaments within the Enlarged European Union: From 'Victims' of Integration to Competitive Actors?, London/New York: Routledge, 2007.

Parijs, Philippe Van. «Justifying Europe», en Luuk van Middelaar y Philippe Van Parijs (2015), After the storm. How to save democracy in Europe, Tielt: Lannoo (2015): 247-262.

Pekonen, Kyosti. «Members of the European Parliament as Delegates and Parliamentarians», en Claudia Wiesner, Kari Palonen y Tapani Turkka (eds.), Parliament and Europe. Rhetorical and conceptual studies on their contemporary connections, Baden-Baden: Nomos, (2011): 59-78.

Piedrafita, Sonia y Blockmans, Steven. Shifting EU Institutional Reform into High Gear: Report of the CEPS High-Level Group, Brussels: Centre for European Policy Studies, 2014.

Pollak, Johannes. «Compounded Representation in the EU: No Country for Old Parliaments?», en Sandra Kröger, (ed), Political Representation in the European Union: Still Democratic in Times of Crisis?, London/New York: Routledge, (2014): 37-69.

Raunio, Tapio y Hix, Simon. «Backbenchers learn to fight back: European integration and parliamentary government»en West European Politics 23/4, (2000): 142-168.

Raunio, Tapio. «National Parliaments and the Future of European Integration: Learning to Play the Multilevel Game», en Achim Hurrelmann, Steffen Schneider y Jens Steffek (eds.), Legitimacy in an Age of Global Politics, New York: Palgrave Macmillan, (2007): 158-173.

Rittberger, Berthold. Building Europe's Parliament. Democratic Representation Beyond the Nation State, Oxford University Press, 2005.

Schmidt, Vivien. "European 'federalism' and its encroachments on national institutions» en Publius 29, N¹, (1999): 19-44.

Schmidt, Vivien. Democracy in Europe. The EU and National Polities, Oxford University Press, 2006.

Shackleton, Michael. "The European Parliament», en John Peterson y Michael Shackleton, The Institutions of the European Union, Oxford University Press, (2012): 124-147.

Sprungk, Carina. «Parlamentarismus in europäischen Mehrebenensystem. Zum Wandel von Rollenanforderungen an nationale Parlamente in EU Angelegenheiten», en Eppler Abels (eds.), Auf dem Weg zum Mehrebenenparlamentarismis? Funktionen 
von Parlamenten im politischen System der EU, Baden-Baden: Nomos, (2011): 211-226.

Thym, Daniel. «Beyond Parliament's Reach? The Role of the European Parliament in the CFSP», European Foreign Affairs, 11, (2006): 109-127.

Tiilikainen, Teija. «The Role of the European Parliament in the EU's Political Order» en Claudia Wiesner, Kari Palonen y Tapani Turkka (eds.), Parliament and Europe. Rhetorical and conceptual studies on their contemporary connections, Baden-Baden: Nomos, 2011, 25-39.

Weiler, Joseph. «In the face of crisis: input legitimacy, output legitimacy and the political messianism of European integration» en Journal of European Integration 34/7, (2012): 825-41. 


\section{Derechos de autor}

Los derechos de autor (para la distribución, comunicación pública, reproducción e inclusión en bases de datos de indexación y repositorios institucionales) de esta publicación (Cuadernos Europeos de Deusto, CED) pertenecen a la editorial Universidad de Deusto. El acceso al contenido digital de cualquier número de Cuadernos Europeos de Deusto es gratuito, transcurridos 6 meses desde su publicación. Los trabajos podrán descargarse, copiar y difundir en cualquier medio sin fines comerciales y según lo previsto por la ley; sin la previa autorización de la Editorial (Universidad de Deusto) o el autor. Así mismo, los trabajos editados en CED pueden ser publicados con posterioridad en otros medios o revistas, siempre que el autor indique con claridad y en la primera nota a pie de página que el trabajo se publicó por primera vez en $C E D$, con indicación del número, año, páginas y DOI (si procede). Cualquier otro uso de su contenido en cualquier medio o formato, ahora conocido o desarrollado en el futuro, requiere el permiso previo por escrito del titular de los derechos de autor.

\section{Copyright}

Copyright (for distribution, public communication, reproduction and inclusion in indexation databases and institutional repositories) of this publication (Cuadernos Europeos de Deusto, CED) belongs to the publisher University of Deusto. Access to the digital content of any Issue of Cuadernos Europeos de Deusto is free only six months after its publication. The content can be accessed, downloaded, copies, and distributed freely in any medium only for non-commercial purposes and in accordance with any applicable copyright legislation, without prior permission from the copyright holder (University of Deusto) or the author. Thus, the content of $C E D$ can be subsequently published in other media or journals, as long as the author clearly indicates in the first footnote that the work was published in $C E D$ for the first time, indicating the Issue number, year, pages, and DOI (if applicable). Any other use of its content in any medium or format, now known or developed in the future, requires prior written permission of the copyright holder. 